\title{
Estructura comunitaria de bivalvos y gasterópodos en raíces del mangle rojo Rhizophora mangle (Rhizophoraceae) en isla Larga, bahía de Mochima, Venezuela
}

\author{
Vanessa Acosta Balbas, Rafael Betancourt Tineo \& Antulio Prieto Arcas \\ Departamento de Biología, Escuela de Ciencias, Universidad de Oriente. Aptdo. 245; Cumaná, 6101. Estado Sucre, \\ Venezuela; vanessaacosta@yahoo.com,rafajose2@gmail.com, aspa2012@hotmail.com
}

\author{
Recibido 18-IX-2012. Corregido 10-III-2013. Aceptado 05-IV-2013.
}

\begin{abstract}
Community structure of bivalves and gastropods in roots of red mangrove Rhizophora mangle (Rhizophoraceae) in isla Larga, Mochima Bay, Venezuela. The Rhizophora mangle roots form a complex ecosystem where a wide range of organisms are permanently established, reproduce, and find refuge. In this study, we assessed the diversity of bivalves and gastropods that inhabit red mangrove roots, in isla Larga, Mochima, Venezuela Sucre state. Bimonthly collections were made from January 2007 to May 2008, in four study areas denominated: South, North, East and West. In each area, five mangrove roots were sampled, and the specimens were obtained. We analyzed a total of 180 roots and a total of 35 bivalve species and 25 gastropod species were found. The most abundant bivalves were: Isognomon alatus, Isognomon bicolor, Ostrea equestris, Crassostrea rhizophorae and Brachidontes exustus; among gastropods, the most common where: Littorina angulifera, Cymatium pileare and Diodora cayenensis. The months with the highest abundances and number of individuals for both groups were January and July 2007, and March 2008. The mangrove ecosystem in isla Larga, presented a number of individuals and species higher than those reported for other regions in Venezuela and the Caribbean. Rev. Biol. Trop. 62 (2): 551-565. Epub 2014 June 01.
\end{abstract}

Key words: Parque Nacional Mochima, red mangrove, roots, molluscs, Venezuela.

Los manglares representan uno de los ecosistemas tropicales y subtropicales más productivos del mundo, ya que conforman subsistemas importantes en estuarios, bahías, ensenadas y lagunas costeras, adaptados a las condiciones de salinidad e inundación propias de un humedal costero y donde además existen procesos biológicos fundamentales para la reproducción, cría y refugio de una gran variedad de invertebrados (FAO, 2007). En estas áreas, los troncos y raíces, particularmente del mangle rojo, son considerados como verdaderas islas de hábitat, porque son frecuentemente circundadas por sedimentos fangosos o arenosos (Kathiresan \& Binghan, 2001). Prácticamente, son los únicos sustratos verticales estables disponibles, constituyendo nichos ecológicos únicos (Coelho, Tenório, Ramos-Porto \& Mello, 2004).
En Venezuela, los manglares cubren aproximadamente $673000 \mathrm{~h}$ a lo largo de toda la costa, en donde las más grandes áreas de manglar están ubicadas en el delta del Orinoco y el golfo de Paria. Existen reportadas seis especies de mangle: Rhizophora mangle, $R$. racemosa, $R$. harrisoni, Laguncularia racemosa, Avicennia germinans y Conocarpus erectus (Conde \& Alarcon, 1993). En nuestro país, se han realizado estudios donde se han caracterizado estos ambientes en diferentes zonas, destacándose los de Medina \& Barboza (2003), Lacerda, Conde, Kjerfve, ÁlvarezLeón, Alarcón \& Polanía (2001), Conde \& Suárez (2003), Barreto (2008), Sánchez-Arias, Paolini \& Rodríguez (2010), López, Barreto \& Conde (2011) y Del Mónaco, Jiménez, Narciso, Alfonso \& Bustillos (2010). Sin embargo, 
desde el punto de vista ecológico, son pocos los trabajos que se han realizado, particularmente sobre organismos asociados a las raíces de $R$. mangle, destacándose los de Morao (1983), Ordosgoitti (1985), Montes (1992), Marcano (1996), Márquez \& Jiménez (2002), Barrios, Márquez \& Jiménez (2003), Márquez-Rojas, Blanco-Rabla, Jiménez \& Allen (2006), Villafranca (2007), Cedeño, Jiménez, Pereda \& Allen (2010), López et al. (2011) y Guerra-Castro, Cruz-Motta \& Conde (2011).

Un componente fundamental de los ecosistemas marinos es el conjunto de especies que constituyen el phylum Mollusca, y representa el mayor contingente en biomasa en lo que a invertebrados se refiere (Prieto, Sant, Méndez \& Lodeiros, 2003; Lee, 2008). Dentro de este grupo hay una gran variedad de organismos sésiles y sedentarios asociados a las raíces de los mangles, de manera que resulta importante conocer sus diferentes grupos taxonómicos, ya que estos pueden actuar como un buen parámetro indicador del ecosistema, considerando que proveen información de las condiciones ambientales de su hábitat tanto por su presencia como por su ausencia. En este sentido, el inventario biológico y el monitoreo en las zonas de manglar, permiten establecer con el tiempo comparaciones con una base de datos, que pueden ser utilizados en cualquier acción de manejo y/o investigación. En este estudio, se evaluó la estructura comunitaria de bivalvos y gasterópodos en raíces del mangle rojo ( $R$. mangle) en isla Larga, ubicada dentro del Parque Nacional Mochima, y su relación con los principales factores ambientales involucrados en este ecosistema. Cabe destacar que los únicos trabajos existentes en isla larga son el de Amaro \& Liñero-Arana (2002), quienes realizaron un inventario de esponjas, mientras que Jiménez \& Liñero-Arana (2002), estudiaron la variación estacional de moluscos en pequeñas praderas de Thalassia testudinum que están alrededor de la isla.

\section{MATERIALES Y MÉTODOS}

Zona de estudio: El Parque Nacional Mochima está ubicado entre $\left(10^{\circ} 9^{\prime} 50^{\prime \prime} \mathrm{y}\right.$ $10^{\circ} 26^{\prime} 0^{\prime \prime} \mathrm{N}$ y $\left.64^{\circ} 13^{\prime} 20^{\prime \prime}-64^{\circ} 47^{\prime} 32^{\prime \prime} \mathrm{W}\right)$, tiene una superficie de 94935 hectáreas, de las cuales un $52 \%$ es superficie marina. Está constituido por acantilados, golfos, costas de aguas profundas, playas de arena blanca, arrecifes de coral, islas e islotes, ensenadas y la bahía de Mochima, que está bordeada por $R$. mangle $\left(10^{\circ} 22^{\prime \prime} 02^{\prime \prime} 10^{\circ} 233^{\prime} 35^{\prime \prime} \mathrm{N}-64^{\circ} 19^{\prime} 38^{\prime \prime} \mathrm{W}\right)$, en la zona sur de la bahía, se encuentra isla Larga $\left(10^{\circ} 21^{\prime} 05^{\prime \prime} 10^{\circ} 21^{\prime} 38 \mathrm{~N}-64^{\circ} 21^{\prime} 05^{\prime}\right.$ - 64'21'25' $\mathrm{W})$, la cual es de pequeñas dimensiones $(500 \mathrm{~m}$ de largo por $200 \mathrm{~m}$ de ancho), circundada en casi toda su extensión por el mangle rojo Rhizophora mangle (Fig. 1).

Metodología empleada: Para conocer la composición taxonómica de la malacofauna establecida en las raíces del mangle rojo, se escogieron cuatro zonas de muestreo, las cuales fueron denominadas según la ubicación geográfica dentro de la isla: sur, norte, este y oeste.

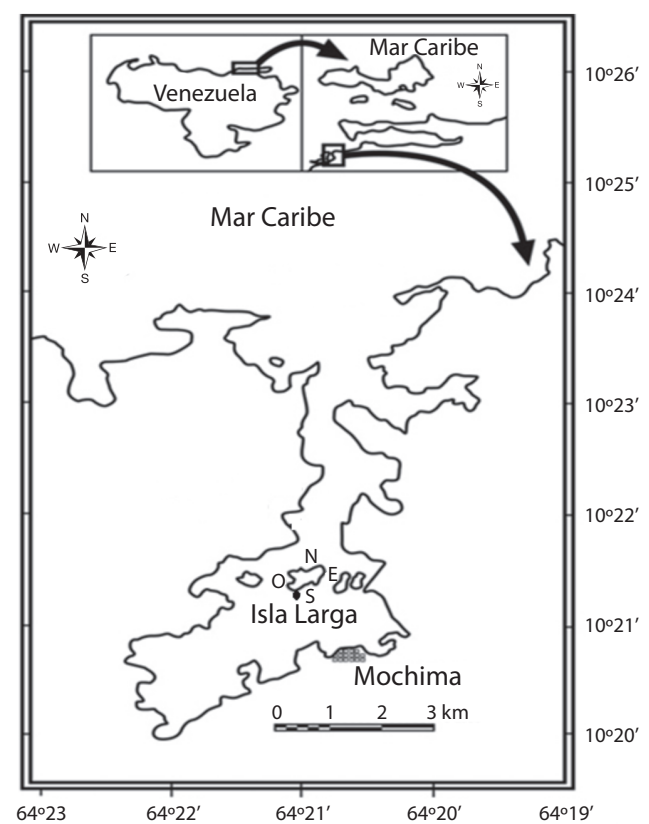

Fig. 1. Mapa de la bahía de Mochima mostrando la ubicación de isla Larga y las zonas de muestreo (norte, sur, este y oeste).

Fig. 1. Map of Mochima bay showing the location of isla Larga and the zones of sampling (North, South, East and West). 
Las zonas norte y este están ubicadas a barlovento y ambas presentan fondos coralinos. En la zona norte, la pendiente del fondo es abrupta y presenta praderas de Thalassia testudinum después de aproximadamente $2 \mathrm{~m}$ de profundidad. Las zonas sur y oeste están situadas a sotavento, donde la pendiente es más moderada, pero con áreas cubiertas de T. testudinum y colonias coralinas dispersas.

Recolección de organismos: Con la finalidad de no afectar el ecosistema durante el tiempo de muestreo, en cada zona se escogieron bimensualmente al azar cinco raíces, desde enero 2007 hasta mayo 2008. Los bivalvos y gasterópodos recolectados fueron depositados en bolsas plásticas previamente etiquetadas. En el laboratorio, se procedió a separar las especies y fueron preservadas en frascos de vidrio con etanol $(70 \%)$. El material fue examinado bajo una lupa estereoscópica binocular, marca Motic, modelo SFC 11. Se emplearon claves de Abbott (1968, 1974), Warmke \& Abbott (1975), Abbott \& Morris (1995), Díaz \& Puyana (1994) y Lodeiros, Marín \& Prieto (1999).

Densidad de organismos: La densidad de los organismos en las raíces se calculó tomando medidas de longitud (largo) y tres medidas de circunferencia a diferentes alturas de cada raíz, con la ayuda de una cinta métrica. Con dichas medidas se aplicó la siguiente fórmula: SM: $\mathrm{L}^{*}(\mathrm{~A} 1+\mathrm{A} 2+\mathrm{A} 3+\ldots . . / \mathrm{AN})$, donde: S.M.=superficie muestreada, $\mathrm{L}=$ longitud de la parte radical muestreada, $\mathrm{A}=$ circunferencia de la raíz a determinada altura, $\mathrm{N}=$ número de medidas de circunferencias tomadas a diferentes alturas. Posteriormente, se procedió a calcular un factor de conversión del área para cada raíz, el cual permitió extrapolar el área muestreada hasta $1 \mathrm{~m}^{2}$. Dicho factor se calculó mediante la relación: $\mathrm{F}=1 \mathrm{~m}^{2} / \mathrm{S} . \mathrm{M}$, con los valores de cada factor de conversión y de los números de organismos recolectados en cada raíz, se procedió a hallar la densidad (org. $/ \mathrm{m}^{2}$ ) según la fórmula: $\mathrm{D}=\mathrm{F}(\mathrm{n})$ x N.O. donde: $\mathrm{D}=$ Densidad (org. $/ \mathrm{m}^{2}$ ), N.O=Número de organismos en cada estación, $\mathrm{F}_{(\mathrm{n})}=$ Factor de conversión en cada una de las estaciones. El cálculo de la densidad es el promedio las cinco raíces por zona de muestreo.

Variables ecológicas: Con el fin de caracterizar la comunidad de moluscos, se determinó la densidad, abundancia mensual y estacional de los organismos y se calcularon los índices de diversidad de Shannon Shannon-Wiener (1963), diversidad de Sanders, equitabilidad de Loyd \& Ghelardi (1964), riqueza específica, constancia por Balgoh (1958) y Bodenheiner (1965), según Krebs (1985), dominancia (McNaughton, 1968) y similaridad de Jaccard (1902) según Margalef (1995). Para conocer el grado de similitud de las diferentes estaciones, según las especies más abundantes se construyó un dendrograma por afinidad, mediante un análisis de conglomerado por el método de centroides, utilizando para ello el programa estadístico computarizado SPSS 10.

Variables ambientales: En cada zona de muestreo, se tomaron muestras de agua superficial para la determinación de los siguientes nutrientes: amonio (Koroleff, 1969), nitrato (Treguer \& Le Corre 1975), nitritos (Bendschneider \& Robinson, 1952), fosfato (Murphy \& Riley, 1962). Al mismo tiempo, en cada estación se midió la temperatura del agua con un termómetro de mercurio de $0.1^{\circ} \mathrm{C}$, el oxígeno disuelto en el agua mediante el método de Winkler, según Strickland \& Parsons (1972) y la salinidad con un refractómetro Portátil, marca Brixco.

Para calcular los componentes comunitarios se utilizó el Programa Ecological Metodology (Krebs, 1989). Para determinar si existen diferencias entre la abundancia y el número de especies por mes y por estación, se realizó mediante un análisis de varianza doble con réplica, previa transformación de los datos ( $\log +1)$, en función de cumplir con las suposiciones de normalidad y homogeneidad de varianzas. En caso de que existan diferencias significativas se aplicó la prueba a posteriori de Duncan (Steel \& Torrie, 1960). 


\section{RESULTADOS}

Se analizaron un total de 180 raíces y se cuantificaron 20893 organismos. La clase Bivalvia estuvo representada por 35 especies pertenecientes a 17 familias, siendo las más abundantes Isognomon alatus, Isognomon bicolor, Ostrea equestris, Crassostrea rhizophorae y Brachidontes exustus, de las familias: Isognomonidae, Ostreidae y Mytiloidae, respectivamente. Para la clase Gasteropoda se obtuvo un total de 25 especies, distribuidas en 14 familias, siendo Littorina angulifera, Cymatium pilearis y Diodora cayenensis, las especies más abundantes durante todo el período de estudio. Las familias Littorinidae, Cymatiidae y Fissurellidae, registraron el mayor número de organismos (Cuadro 1).

La densidad de organismos en las raíces mostró diferencias significativas entre las zonas y los meses ( $\mathrm{Fs}=22.70 ; \mathrm{p}<0.05$; $\mathrm{Fs}=23.54 ; \mathrm{p}<0.001$ ), las mayores densidades se registraron en las zonas sur (507.02 org./ $\mathrm{m}^{2}$ ) y oeste $\left(290.23 \mathrm{org} . / \mathrm{m}^{2}\right)$, y las menores en las zonas norte $\left(154.66 \mathrm{org} . / \mathrm{m}^{2}\right)$ y este $(173.60$ org. $/ \mathrm{m}^{2}$ ), respectivamente (Fig. 2A). En enero, julio y marzo, se reportaron las mayores densidades, mientras que los valores más bajos se reportaron en octubre y noviembre (Fig. 2B).

Con respecto a la abundancia se obtuvieron diferencias significativas entre las estaciones $(F s=5.32 ; p<0.001)$, mientras que entre los meses no se encontraron diferencias $(\mathrm{Fs}=3.82$; $\mathrm{p}>0.05)$. Los mayores valores se encontraron en la zona sur (11 029 ind.), seguida por la zona oeste (4 788 ind.), mientras que los valores más bajos se obtuvieron en la zona norte (2 491 ind.) y este (2 585 ind.), respectivamente (Cuadro 2). En la figura 3, se muestra la variación mensual de la abundancia, los máximos valores se registraron en enero, julio y marzo, mientras que las menores abundancias en septiembre y noviembre. De las 60 especies de moluscos recolectadas en este estudio, 22 resultaron ser constantes en todas las zonas, se encontraron en el $100 \%$ de las raíces, otras 11 especies se observaron en un $75 \%$ de las raíces. Por su parte, 15 especies fueron consideradas accesorias ya que se hallaron en el 50\% de las raíces analizadas, mientras que tan solo 12 especies fueron consideradas raras o accidentales, ya que estuvieron presentes en menos del 25\% (Cuadro 2).

\section{CUADRO 1}

Clasificación taxonómica de las especies de moluscos colectadas en las raíces del mangle rojo (Rhizophora mangle), en las diferentes zonas de muestreo (sur, norte, este y oeste) de isla Larga, bahía de Mochima, estado Sucre, Venezuela

TABLE 1

Taxonomic classification of the shellfish species collected in the roots of red mangrove (Rhizophora mangle), in the different sampling zones (South, North, East and West) of Long island, Mochima bay, Sucre state, Venezuela

\begin{tabular}{|c|c|c|c|c|}
\hline Phylum & Clase & Orden & Familia & Especie \\
\hline \multirow[t]{13}{*}{ Mollusca } & Bivalvia & Nuculoida & Nuculanidae & Nuculana acuta (Conrad, 1931) \\
\hline & & Arcoida & Arcidae & Arca imbricata (Bruguière, 1789) \\
\hline & & & & Arca zebra (Swainson, 1833) \\
\hline & & & & Barbatia candida (Helbing, 1799) \\
\hline & & & & Anadara notabilis (Röding, 1798) \\
\hline & & Mytiloida & Mytilidae & Brachidontes exustus (Linnè, 1758) \\
\hline & & & & Ryenella lateralis (Say, 1821) \\
\hline & & & & Lithophaga bisulcata (d Orbigny, 1842) \\
\hline & & & & Brachidontes domingensis (Lamarck, 1819) \\
\hline & & & & Lithophaga aristata (Dillwyn, 1817) \\
\hline & & & & Modiolus squamosus (Beauperthuy, 1967) \\
\hline & & Pteroida & Pteriidae & Pteria colymbus (Röding, 1798) \\
\hline & & & & Pinctada imbricata (Röding, 1798) \\
\hline
\end{tabular}


CUADRO 1 (Continuación) / TABLE 1 (Continued)

\begin{tabular}{|c|c|c|c|c|}
\hline Phylum & Clase & Orden & Familia & Especie \\
\hline & & & Isognomonidae & Isognomon alatus (Gmelin, 1791) \\
\hline & & & & Isognomon bicolor (C.B Adams, 1845) \\
\hline & & & & Isognomon radiatus (Aton, 1839) \\
\hline & & & Malleidae & Malleus candeanus (d Orbingny, 1842) \\
\hline & & & Ostreidae & Ostrea equestris (Say, 1834) \\
\hline & & & & Crassostrea rhizophorae (Guilding, 1828) \\
\hline & & & & Crassostrea virginica (Gmeling, 1791) \\
\hline & & & & Lopha frons (Linnè, 1758) \\
\hline & & & Plicatulidae & Plicatula gibbosa (Lamarck, 1801) \\
\hline & & & Spondylidae & Spondylus americanus (Hermann, 1781) \\
\hline & & & Limidae & Lima scabra (Born, 1778) \\
\hline & & & Anomiidae & Anomia ephippiun (Linnè, 1758) \\
\hline & & Veneroida & Pectinidae & Lyropecten nodosus (Linnè, 1758) \\
\hline & & & Luciidae & Codakia orbicularis (Linnè, 1758) \\
\hline & & & Chamidae & Chama macerophylla (Gmelin, 1791) \\
\hline & & & & Chama congregata (Conrad, 1833) \\
\hline & & & Cardiidae & Papyridea soleniformis (Bruguière, 1789) \\
\hline & & & & Trachycardium muricatum (Linnè, 1758) \\
\hline & & & & Microcardium tinctum (Dall, 1881) \\
\hline & & & Veneridae & Chione intapurpurea (Conard, 1849) \\
\hline \multirow[t]{25}{*}{ Gasterópoda } & Archeogastropoa & & Fissurellidae & Hemitoma octoradiata (Gmelin, 1791) \\
\hline & & & & Diodora cayennensis (Lamarck, 1822) \\
\hline & & & & Diodora minuta (Lamarck, 1822) \\
\hline & & & & Fissurella nimbosa (Linnè, 1758) \\
\hline & & & & Diodora dysoni (Reeve, 1850) \\
\hline & & & & Fissurella barbadensis (Gmelin, 1791) \\
\hline & & & Turbinidae & Turbo castanea (Gmelin, 1791) \\
\hline & & & & Astraea phoebia (Röding, 1798 \\
\hline & & & Trochidae & Tegula fasciata (Born, 1778) \\
\hline & & & Acmaeidae & Acmaea antillarum (Sowerby, 1931) \\
\hline & & & Neritidae & Nerita fulgurans (Gmelin, 1791) \\
\hline & & & & Nerita tessellata (Gmelin, 1791) \\
\hline & & Mesogastropoda & Littorinidae & Littorina nebulosa (Lamarck, 1822) \\
\hline & & & & Littorina angulifera (Lamarck, 1822) \\
\hline & & & & Littorina tessellata (Lamarck, 1822) \\
\hline & & & Cerithidae & Cerithium litteratun (Born, 1778) \\
\hline & & & & Cerithium eburneum (Bruguiere, 1792) \\
\hline & & & Potamididae & Batillaria minina (Gmelin, 1791) \\
\hline & & & Turritellidae & Turritella variegata (Linnè, 1758) \\
\hline & & & Thaididae & Thais haemastoma (Conrad, 1837) \\
\hline & & & Cymatiidae & Cymatium (Septa) pileare (Linnè, 1758) \\
\hline & & Neogasterópoda & Muricidae & Murex recurvirostrus (Conarad, 1837) \\
\hline & & & & Chicoreus brevifrons (Lamarck, 1822) \\
\hline & & & Fasciolariidae & Leucozonia nassa (Gmelin, 1791) \\
\hline & & Basommatophara & Melampidae & Melampus coffeus (Linnè, 1758) \\
\hline
\end{tabular}



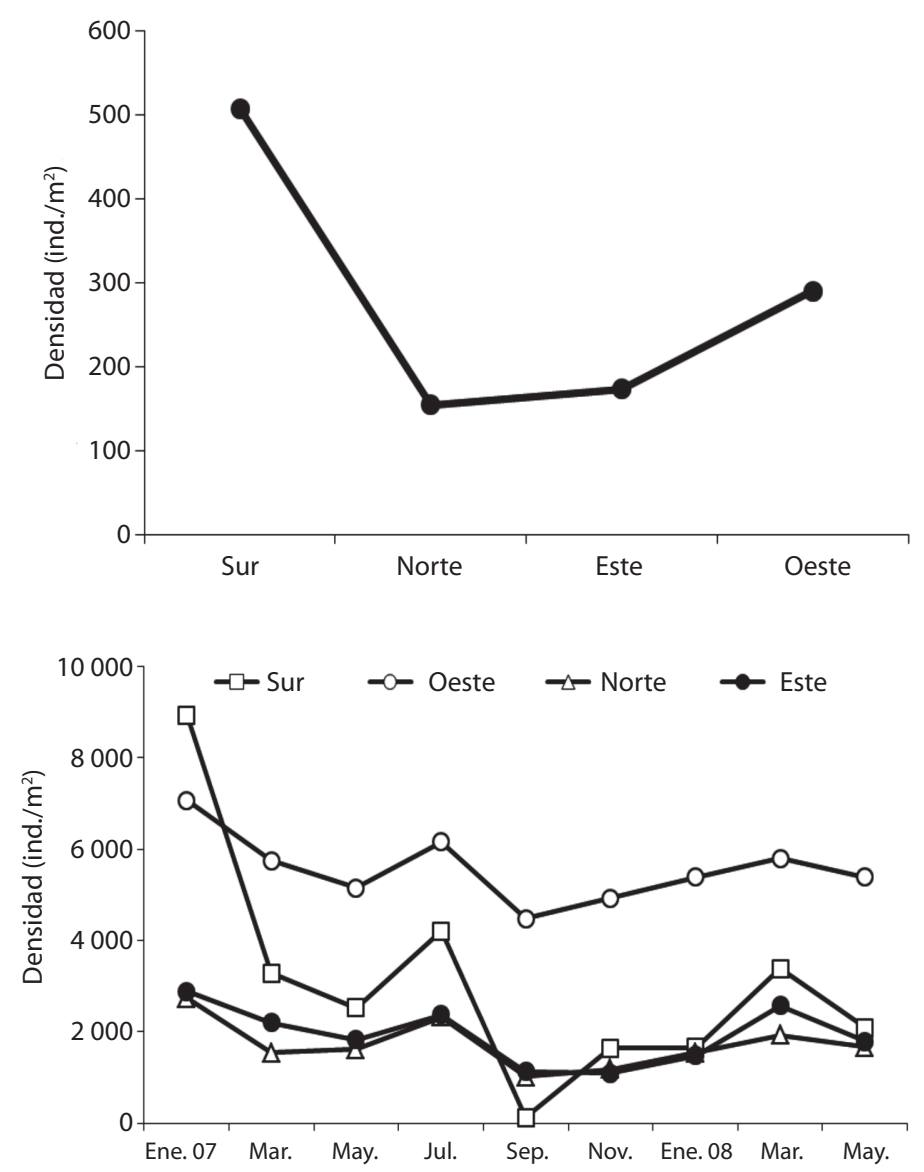

Fig. 2. (A) Variación zonal y (B) mensual de la densidad (ind. $/ \mathrm{m}^{2}$ ) en las raíces del mangle rojo (Rhizophora mangle), de isla Larga, bahía de Mochima, estado Sucre, Venezuela.

Fig. 2. (A) Variation zonal and (B) density monthly (ind. $/ \mathrm{m}^{2}$ ) in roots of red mangrove (Rhizophora mangle), isla Larga, bay Mochima, Sucre state, Venezuela.

El índice de diversidad fue similar en cada una de las zonas estudiadas $(\mathrm{Fs}=\mathrm{p}>0.05$; 4.52), mientras que mensualmente sí hubo una variabilidad significativa $(\mathrm{Fs}=8.73 ; \mathrm{p}<0.05)$ (Fig. 4). La equitabilidad por estación mantuvo una correspondencia con la diversidad, el valor más alto correspondió a la zona norte y el más bajo a la zona sur. Los valores de riqueza en cada una de las zonas de muestreo no correspondieron con la diversidad, la riqueza más alta se encontró en la zona sur y la menor en la zona norte, así mismo los mayores registros se obtuvieron en enero y julio 2007 y marzo 2008 (Fig. 4B). El índice de rarefación de
Sanders, estimado para 1000 individuos, varió entre 13-27 especies (Cuadro 3). El índice de dominancia porcentual mostró que los bivalvos fueron más dominantes que los gasterópodos, las especies más dominantes de ambos grupos fueron: Isognomon alatus (47.91\%), Isognomon bicolor (17.53\%), Ostrea equestris (16.53\%), Brachidontes exustus (14.51\%) y Crassostrea rhizophorae (12.38\%), Littorina angulifera (2.58\%), Cymatium pileare (1.48) y Diodora cayenensis $(1.23 \%)$.

El dendrograma de similitud, según la presencia de especies en las diferentes zonas muestreadas, muestra la existencia de dos 


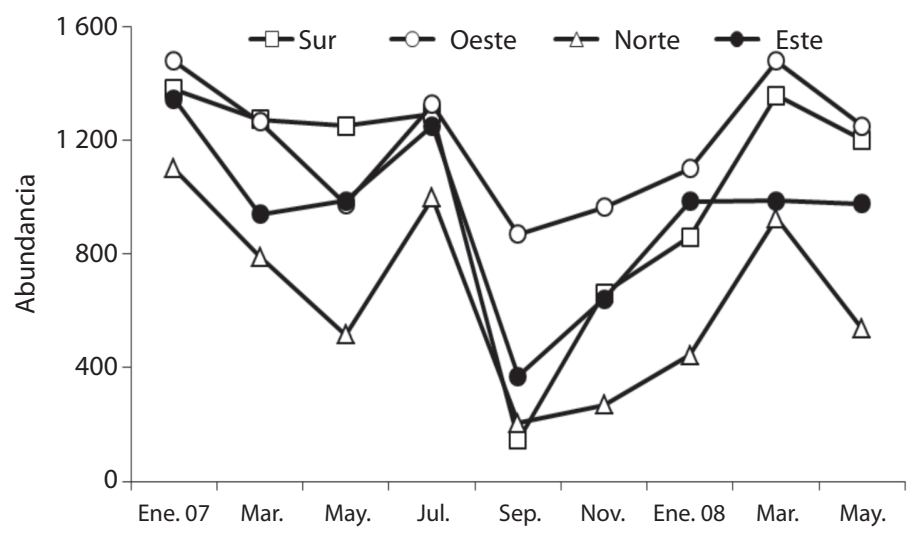

Fig. 3. Variación mensual de la abundancia de organismos en las raíces del mangle rojo (Rhizophora mangle) en las diferentes zonas de muestreo de isla Larga, Bahía de Mochima, estado Sucre, Venezuela.

Fig. 3. Monthly variation of abundance of organisms in the roots of red mangrove (Rhizophora mangle) in different sampling zones of isla Larga, Mochima bay, Sucre state, Venezuela.

\section{CUADRO 2}

Número de organismos por especie contabilizados indicando su presencia o ausencia (-) e índice de constancia recolectadas en las diferentes zonas de muestreo.

TABLE 2

Number of organisms accounted for by species indicating their presence or absence (-) and index of evidence collected in the different sampling areas.

\begin{tabular}{lcccccc}
\multicolumn{1}{c}{ Especies } & Zona sur & Zona norte & Zona este & Zona oeste & Total & Índice de Constancia \\
Isognomon alatus & 4631 & 736 & 923 & 1806 & 8096 & C $(100 \%)$ \\
Lithophaga aristata & 36 & 2 & - & 8 & 46 & C $(75 \%)$ \\
Brachidontes exustus & 777 & 137 & 434 & 330 & 1678 & C $(100 \%)$ \\
Ostrea equestris & 1069 & 122 & 160 & 387 & 1738 & C $(100 \%)$ \\
Arca imbricata & 34 & 2 & 1 & 35 & 72 & C $(100 \%)$ \\
Isognomon bicolor & 831 & 435 & 141 & 524 & 1931 & C $(100 \%)$ \\
Crassostrea rhizophorae & 905 & 64 & 170 & 220 & 1359 & C $(100 \%)$ \\
Isognomon radiatus & 239 & 113 & 262 & 569 & 1183 & C $(100 \%)$ \\
Arca zebra & 14 & 3 & 2 & 14 & 33 & C $(100 \%)$ \\
Chama macerophylla & 251 & 5 & 10 & 34 & 300 & C $(100 \%)$ \\
Pinctada imbricata & 587 & 161 & 166 & 319 & 1233 & C $(100 \%)$ \\
Crassostrea virginica & - & 1 & - & - & 1 & Acc $(25 \%)$ \\
Pteria Columbus & 197 & 12 & 49 & 36 & 294 & C $(100 \%)$ \\
Spondylus americanus & 210 & 2 & 24 & 43 & 279 & C $(100 \%)$ \\
Brachidontes domingensis & 311 & 89 & 68 & 137 & 605 & C $(100 \%)$ \\
Lithophaga bisulcata & 210 & - & 2 & 2 & 214 & C $(75 \%)$ \\
Lopha frons & 3 & 79 & - & - & 82 & Acs $(50 \%)$ \\
Nerita fulgurans & 3 & 67 & - & - & 70 & Acs $(50 \%)$ \\
Lima scabra & 4 & - & - & - & 4 & Acc $(25 \%)$ \\
Chione intapurpurea & 2 & 1 & - & - & 3 & Acs $(50 \%)$ \\
Trachycardium muricatum & 2 & 1 & - & - & 3 & Acs $(50 \%)$ \\
Chicoreus brevifrons & - & 17 & - & - & 17 & Acs $(50 \%)$ \\
Littorina angulifera & 118 & 101 & 39 & 108 & 366 & C $(100 \%)$
\end{tabular}


CUADRO 2 (Continuación) / TABLE 2 (Continued)

\begin{tabular}{|c|c|c|c|c|c|c|}
\hline Especies & Zona sur & Zona norte & Zona este & Zona oeste & Total & Índice de Constancia \\
\hline Diodora cayenensis & 71 & 25 & 16 & 24 & 136 & $\mathrm{C}(100 \%)$ \\
\hline Cymatium pileare & 92 & 33 & 14 & 35 & 174 & C $(100 \%)$ \\
\hline Diodora minuta & 57 & 9 & 8 & 47 & 121 & C $(100 \%)$ \\
\hline Turbo castanea & - & 10 & - & 6 & 16 & $\operatorname{Acs}(50 \%)$ \\
\hline Littorina tessellata & 34 & 184 & 14 & 6 & 238 & $\mathrm{C}(100 \%)$ \\
\hline Littorina nebulosa & 7 & 44 & - & - & 51 & $\operatorname{Acs}(50 \%)$ \\
\hline Murex brevifrons & 28 & - & 22 & 4 & 54 & $\mathrm{C}(75 \%)$ \\
\hline Anadara notabilis & 1 & - & - & 2 & 3 & $\operatorname{Acs}(50 \%)$ \\
\hline Diodora dysonii & 13 & 6 & 4 & 10 & 33 & C (100\%) \\
\hline Lyropecten nodosus & 15 & - & - & - & 15 & $\operatorname{Acc}(25 \%)$ \\
\hline Ryenella lateralis & 9 & - & - & 3 & 12 & $\operatorname{Acs}(50 \%)$ \\
\hline Papyridea soleniformis & 8 & - & 6 & - & 14 & $\operatorname{Acs}(50 \%)$ \\
\hline Batillaria minina & 10 & 7 & - & - & 17 & $\operatorname{Acs}(50 \%)$ \\
\hline Chama congregata & 33 & - & 1 & 22 & 56 & $\mathrm{C}(75 \%)$ \\
\hline Microcardium tinctum & 2 & - & - & - & 2 & $\operatorname{Acc}(25 \%)$ \\
\hline Thais haemastoma & 6 & - & - & - & 6 & $\operatorname{Acc}(25 \%)$ \\
\hline Glycymeris pectinata & 3 & - & - & - & 3 & $\operatorname{Acc}(25 \%)$ \\
\hline Modiolus squamosus & 8 & - & - & - & 8 & $\operatorname{Acc}(25 \%)$ \\
\hline Turritella variegata & 4 & - & - & - & 4 & $\operatorname{Acc}(25 \%)$ \\
\hline Nuculana acuta & - & 2 & - & - & 2 & $\operatorname{Acc}(25 \%)$ \\
\hline Barbatia candida & 8 & - & - & - & 8 & $\operatorname{Acc}(25 \%)$ \\
\hline Malleus candeanus & 1 & - & - & - & 1 & $\operatorname{Acc}(25 \%)$ \\
\hline Melampus coffeus & 3 & - & - & - & 3 & $\operatorname{Acc}(25 \%)$ \\
\hline Acmaea antillarum & 38 & 3 & - & 19 & 60 & C $(100 \%)$ \\
\hline Plicatula gibbosa & 51 & - & 2 & 9 & 62 & $\mathrm{C}(75 \%)$ \\
\hline Perna perna & 2 & - & - & - & 2 & $\operatorname{Acc}(25 \%)$ \\
\hline Anomia ephippium & 43 & - & 6 & 14 & 63 & $\mathrm{C}(75 \%)$ \\
\hline Nerita tessellata & - & 13 & - & - & 13 & $\operatorname{Acc}(25 \%)$ \\
\hline Astrea phoebia & 6 & - & 6 & 2 & 14 & $\mathrm{C}(75 \%)$ \\
\hline Cerithium litteratum & 11 & 2 & 5 & - & 18 & $\mathrm{C}(75 \%)$ \\
\hline Cerithium eburneum & 2 & - & 8 & - & 10 & $\operatorname{Acs}(50 \%)$ \\
\hline Tegula fasciata & 2 & - & 9 & - & 11 & $\operatorname{Acs}(50 \%)$ \\
\hline Hemitoma octoradiata & 10 & 2 & 1 & 4 & 17 & $\mathrm{C}(100 \%)$ \\
\hline Fissurella nimbosa & 7 & - & 3 & 3 & 13 & $\mathrm{C}(75 \%)$ \\
\hline Fissurella barbadensis & 1 & 1 & 1 & 3 & 6 & $\mathrm{C}(75 \%)$ \\
\hline Leucozonia nassa & 2 & 1 & 6 & - & 9 & $\mathrm{C}(75 \%)$ \\
\hline Codakia orbicularis & 5 & 1 & 2 & 3 & 11 & $\mathrm{C}(100 \%)$ \\
\hline TOTAL & 11029 & 2491 & 2585 & 4788 & 893 & \\
\hline
\end{tabular}

C: Constantes, Acs: Accesoria: Acc: Accidental.

C: Constants, Acs: Accessory: Acc: Accidental.

grupos, el primer grupo constituido por la zona norte, mientras que el segundo grupo está formado por las zonas este, oeste y sur, respectivamente (Fig. 5).

La temperatura mensual entre las zonas fluctuó de $23^{\circ} \mathrm{C}$ a $29^{\circ} \mathrm{C}$, se observaron valores más altos entre julio y septiembre 2007, donde la temperatura osciló entre 28 y $29^{\circ} \mathrm{C}$, para luego disminuir en enero y marzo 2008, la cual se mantuvo en $22^{\circ} \mathrm{C}$. Este parámetro mostró un promedio anual de $25 \pm 2.45^{\circ} \mathrm{C}$. La salinidad se mantuvo casi constante, se obtuvieron registros 


\section{CUADRO 3}

Índices ecológicos de la comunidad de moluscos bivalvos y gasterópodos presentes en las diferentes zonas de muestreo de isla Larga, bahía de Mochima, estado Sucre, Venezuela

TABLE 3

Ecological indices community bivalves and gastropods present in the different sampling zones of Long island, Mochima bay, Sucre state, Venezuela

\begin{tabular}{lcccc} 
& Zona sur & Zona oeste & Zona norte & Zona este \\
Riqueza (S') & 56 & 36 & 33 & 34 \\
Diversidad (H') & 3.23 & 3.16 & 3.23 & 3.15 \\
Diversidad Sanders & 54 & 35 & 32 & 33 \\
Equitabilidad & 0.55 & 0.68 & 0.55 & 0.62 \\
Diversidad Simpon (1-D) & 0.98 & 0.97 & 0.98 & 0.97 \\
\hline
\end{tabular}
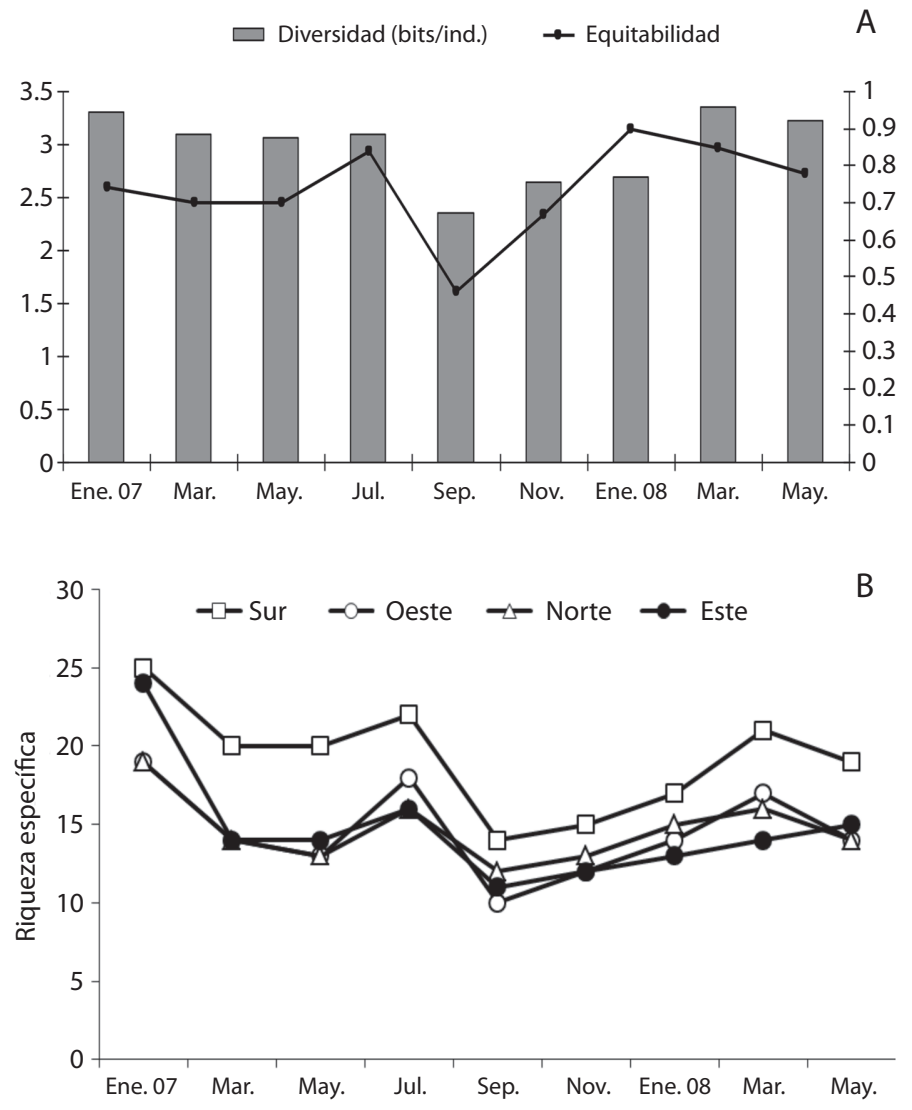

Fig. 4. (A) Variación mensual de la diversidad (bits/ind.) y equitabilidad y (B) riqueza en las diferentes zonas de muestreo de isla Larga, bahía de Mochima, estado Sucre, Venezuela.

Fig. 4. (A) Monthly variation of diversity (bits/ind.) and equitability and (B) richness in the different sampling zones of isla Larga, Mochima bay, Sucre state, Venezuela. 


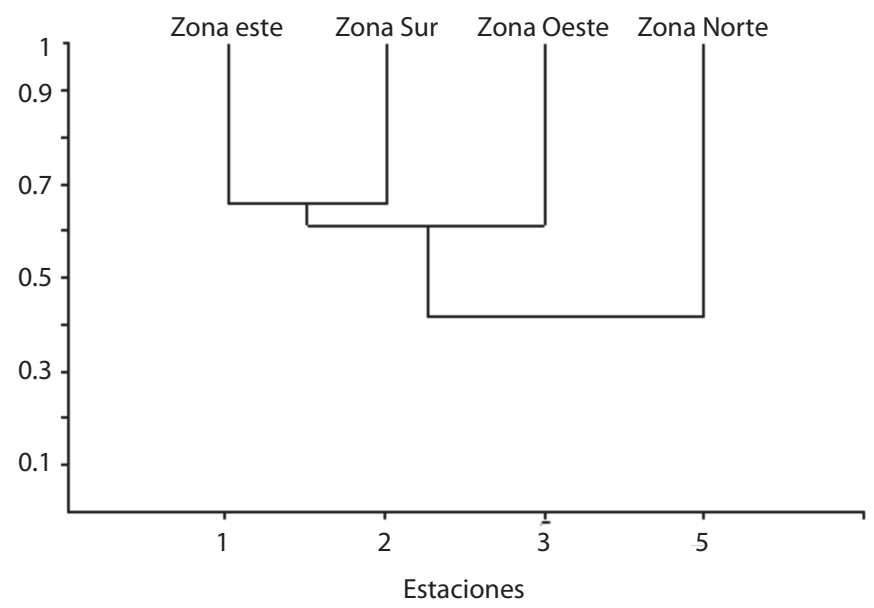

Fig. 5. Dendrograma por similitud de las diferentes zonas de muestreo (sur, norte, este y oeste) de isla Larga, bahía de Mochima, estado Sucre, Venezuela.

Fig. 5. Dendrogram of similarity of different sampling areas (South, North, East and West) of isla Larga, Bay Mochima, Sucre state, Venezuela.

entre 35-38psu, los más altos valores se presentaron en noviembre 2007 ( $>37 \mathrm{psu}$ ), mientras que los más bajos (35psu) se registraron en enero 2007 y enero 2008, con promedio de $37 \pm 1.16 \mathrm{psu}$. Las concentraciones de oxígeno en el agua también presentaron poca variación,

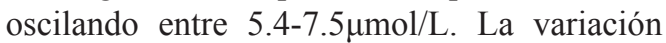
mensual del oxígeno disuelto fue muy similar a la temperatura, en la época de temperaturas bajas se presentaron las concentraciones más bajas de oxígeno, con máximos valores en mayo y noviembre 2007 y los menores en enero, marzo 2007 y enero 2008, lo que coincide con las bajas temperaturas relativas de la época de surgencia. El promedio de los niveles de oxígeno obtenido en todas las estaciones fue de $6.24 \pm 0.07 \mathrm{mg} / \mathrm{mL}$ (Cuadro 4).

El nitrato osciló entre 0.45 y $1.24 \mu \mathrm{mol} / \mathrm{L}$ y los máximos valores de nitrato se registraron en enero, julio y marzo, mientras que valores más bajos se obtuvieron en septiembre, noviembre y mayo. La media fue de $0.87 \pm 0.34 \mu \mathrm{mol} / \mathrm{L}$. Los niveles mensuales de amonio fluctuaron entre $0.55-1.68 \mu \mathrm{mol} / \mathrm{L}$, alcanzando sus valores más altos en noviembre y mayo y los más bajos en julio y marzo, la media fue de $1.06 \pm 0.37 \mu \mathrm{mol} / \mathrm{L}$. Las concentraciones de fósforo mostraron variaciones a lo largo de todo el año, los máximos valores se registraron en enero, julio y marzo, y el mínimo en septiembre, el promedio total fue de $1.03 \pm 0.62 \mu \mathrm{mol} / \mathrm{L}$ (Cuadro 4).

\section{DISCUSIÓN}

El número de especies de moluscos (60) asociados a las raíces de mangle en isla Larga, fue elevado con respecto a otros trabajos realizados en el Nororiente de Venezuela (Morao, 1983; Odrosgoitti, 1985; Márquez \& Jiménez, 2002; Cedeño, Jiménez, Pereda \& Allen, 2010) así como en otras zonas del Caribe (Murillo, 2004; Portocarrero, 2004; Pomareda \& Zanella, 2006; Moreno-Ríos, 2007; Valle, 2007; Pico, Hernández-Rivas \& Holguín-Quiñones, 2008). Los altos valores reportados en este estudio, pueden deberse a las características de la isla, que presenta una mezcla de diferentes ambientes que proporcionan la existencia de una cantidad de nichos que actúan como zonas de refugio y protección para el establecimiento de muchos organismos, tomando en consideración que isla Larga está ubicada dentro de un Parque Nacional. 
CUADRO 4

Valores promedios mensuales $(*)$ de los parámetros ambientales registrados en isla Larga, bahía de Mochima, estado Sucre, Venezuela.

TABLE 4

Monthly mean values (*) of environmental parameters recorded in Long island, Mochima bay, Sucre state, Venezuela.

\begin{tabular}{|c|c|c|c|c|c|c|}
\hline Meses & $\begin{array}{c}\text { Nitrato } \\
(\mu \mathrm{mol} / \mathrm{L})\end{array}$ & $\begin{array}{l}\text { Fosfato } \\
(\mu \mathrm{mol} / \mathrm{L})\end{array}$ & $\begin{array}{l}\text { Amonio } \\
(\mu \mathrm{mol} / \mathrm{L})\end{array}$ & Temperatura $\left({ }^{\circ} \mathrm{C}\right)$ & Salinidad (PSU) & $\begin{array}{l}\text { Oxigeno } \\
(\mathrm{mg} / \mathrm{mL})\end{array}$ \\
\hline Ene-07 & $1.24 \pm 0.17$ & $1.49 \pm 0.26$ & $1.03 \pm 0.14$ & $23 \pm 0.25$ & $35 \pm 0.56$ & $5.43 \pm 0.28$ \\
\hline Mar & $1.15 \pm 0.23$ & $0.14 \pm 0.09$ & $1.07 \pm 0.17$ & $23 \pm 0.83$ & $37 \pm 0.43$ & $6.22 \pm 0.06$ \\
\hline May & $0.59 \pm 0.21$ & $0.92 \pm 0.40$ & $1.16 \pm 0.40$ & $27 \pm 0.43$ & $37 \pm 0.43$ & $7.45 \pm 0.34$ \\
\hline Jul & $1.43 \pm 1.18$ & $1.42 \pm 0.20$ & $0.75 \pm 0.45$ & $28 \pm 0.29$ & $37 \pm 0.43$ & $6.60 \pm 0.25$ \\
\hline Sep & $0.59 \pm 0.20$ & $0.62 \pm 0.13$ & $1.28 \pm 0.27$ & $29 \pm 0.40$ & $37 \pm 0.50$ & $6.66 \pm 0.26$ \\
\hline Nov & $0.64 \pm 0.13$ & $0.18 \pm 0.12$ & $1.42 \pm 0.21$ & $27 \pm 0.30$ & $38 \pm 0.01$ & $6.82 \pm 0.17$ \\
\hline Ene-08 & $0.98 \pm 0.16$ & $1.38 \pm 0.10$ & $0.55 \pm 0.22$ & $22 \pm 0.10$ & $35 \pm 0.54$ & $5.60 \pm 0.25$ \\
\hline Mar & $0.82 \pm 0.12$ & $1.98 \pm 0.29$ & $0.64 \pm 0.16$ & $22 \pm 0.53$ & $38 \pm 0.43$ & $6.57 \pm 0.24$ \\
\hline May & $0.45 \pm 0.09$ & $1.17 \pm 0.07$ & $1.68 \pm 0.13$ & $26 \pm 0.62$ & $38 \pm 0.43$ & $6.65 \pm 0.20$ \\
\hline Prom. Total & $0.87 \pm 0.34$ & $1.03 \pm 0.62$ & $1.06 \pm 0.37$ & $25 \pm 2.45$ & $37 \pm 1.16$ & $6.24 \pm 0.07$ \\
\hline
\end{tabular}

(*)=Promedio \pm desviación estándar.

Las especies más abundantes fueron los bivalvos: I. alatus, I. bicolor, O. equestris, y $B$. exustus y $C$. rhizophorae, mientras que dentro de los gasterópodos destacaron L. angulifera, C. pileare y D. cayenensis. Dichas especies también han sido señaladas como abundantes en otras zonas de manglar en diferentes regiones del mar Caribe como en el golfo de Santa Fe: I. alatus, I. bicolor, O. equestris, $B$. exustus, $C$. rhizophorae, Littorina meleagris y L. aristata (Márquez \& Jiménez, 2002). En el caribe Colombiano: I. alatus, B. exustus y $C$. rhizophorae, L. angulifera, C. pileare y $D$. cayenensis, son las especies características de las raíces sumergidas del mangle rojo en Isla Barú (Portocarrero 2004), en la Ciénaga de Cholón: Brachidontes exustus e Isognomon alatus, muestran una amplia distribución y abundancia (Valle, 2007). En el ambiente de manglar de Ensenada la Paz, México las especies más abundantes son: Crassostrea palmula, Anadara tuberculosa y Brachidontes semilaevis (Pico et al., 2008). La mayoría de las especies citadas anteriormente, han sido catalogadas como fauna característica de los ecosistemas de manglar, ya que necesitan de un sustrato duro para fijarse (particularmente en el caso de los moluscos).

La mayor abundancia y densidad de organismos en las raíces de mangle rojo correspondió a las zonas sur y oeste, lo cual podría estar asociado a las áreas coralinas y parches de $T$. testudinum cercanas a dichas zonas, que suministra especies gracias al transporte (migración) hacia los manglares. Prieto et al. (2003), reportaron en parches de T. testudinum, cercanas a isla Larga, la presencia de 41 especies de gasterópodos y 40 de bivalvos. El hecho de que estas zonas estén situadas en áreas protegidas (sotavento), permite un abastecimiento constante de nutrientes, indispensables para los organismos filtradores, especialmente los bivalvos. En las zonas norte y este, sin embargo, se obtuvieron los valores más bajos de abundancia y de densidad por raíz, debido por un lado a la escasa presencia y poco desarrollo del mangle y por estar alejados de las zonas rocosas, coralinas y de praderas de T. testudimun. Otro factor que probablemente limita la presencia de organismos en la zona norte, es la dinámica costera de la zona como producto de una mayor incidencia de los vientos (zona de barlovento), el 
cual origina un mayor oleaje, lo que impide el establecimiento de organismos en sus etapas larvarias en las raíces de mangle. Por su parte, la zona este, está expuesta a la circulación permanente de embarcaciones procedentes desde el pueblo de Mochima hacia las diferentes playas turísticas de la zona, hecho que puede estar influyendo negativamente sobre la fijación de organismos. Se sugiere hacer un estudio que permita corroborar la hipótesis antes señalada en donde se estudie la interacción de arrecifes con pastos marinos y manglares en isla Larga.

Los picos de máxima abundancia de organismos por raíz, estuvieron relacionados con el período de mayor intensidad de la surgencia costera que acontece en la zona entre los meses de diciembre a abril y mayo, la cual viene acompañada de bajas temperaturas, salinidad alta, baja concentración de oxígeno y altas concentraciones de nutrientes (fosfatos y nitratos). No obstante, la abundancia de organismos obtenida en julio pudiera estar asociada con el segundo período de surgencia, que ocurre entre los meses de julio y agosto, que es de menor intensidad que la que se produce durante los primeros meses del año (Okuda, 1975; Herrera \& Febres, 1975; Quintero et al., 2004).

En líneas generales, la diversidad registrada en este estudio puede considerarse alta, si se compara con otros trabajos, donde se tomaron en cuenta diferentes filos de organismos asociados a las raíces de mangle. En este sentido, Ordosgoitti (1985) en la bahía de Mochima reportó una diversidad entre 1.06 y $2.44 \mathrm{bits} /$ ind., Márquez \& Jiménez (2002) hallaron valores entre 2.72 y 3.19 bits/ind., en el Golfo de Santa Fe, Villafranca (2007) reportó en la laguna de La Restinga valores entre 2.49 y 3.0bits/ ind., Cedeño et al. (2010) encontró una diversidad de 3.17 y 4.86 bits/ind., en la laguna de Bocaripo. En el Caribe, particularmente en las costas colombianas, Valle (2007) registró una diversidad que osciló entre 2.33 y 2.81 bits/ind., en la Ciénaga de Cholón, isla Barú.

La diversidad mostró poca variación mensual se mantuvo por encima de $2.5 \mathrm{bits} / \mathrm{ind}$., durante casi todo el año, a excepción de septiembre y noviembre donde cae, asociada posiblemente con periodos reproductivos de las especies. La diversidad en la zona pudo estar relacionada, posiblemente, con el comportamiento reproductivo de los bivalvos y gasterópodos, los cuales se caracterizan por tener un tipo de reproducción continua, típica de especies tropicales, en donde el ambiente, en general, no juega un papel significativo en la regulación de su ciclo reproductivo, siendo en general los factores endógenos los reguladores de la reproducción, lo que explicaría la presencia de organismos durante todo el año de estudio. Los altos valores de diversidad, con respecto a otros trabajos realizados en las áreas del Caribe y otras regiones de Venezuela podrían ser explicados por varias razones: a) La bahía de Mochima muestra características hidroquímicas que influyen positivamente sobre la biota marina presente en la zona, b) la distribución y cobertura de los bosques de mangle en isla Larga, unido a la proximidad de hábitats circundantes (zonas rocosas, coralinas y praderas de fanerógamas marinas), benefician de manera significativa la presencia de organismos en las raíces de mangle y c) el tipo de muestreo realizado, el cual fue de manera continua durante nueve meses, además de lo antes expuesto, el estudio fue realizado en un área que se encuentra dentro de un parque nacional.

Cabe destacar que la zona sur, a pesar de no mostrar la diversidad más alta, presentó la mayor riqueza y el mayor número de individuos, lo cual podría estar asociado a la alta presencia de I. alatus, que aportó la mayor cantidad de organismos, dominando en muchos casos toda el área de las raíces y actuando de esta manera como especie constructora de hábitat, sirviendo de sustrato para la fijación de otros organismos. Por otro lado, I. alatus es considerada una especie exótica e invasora en zonas como Brasil (Domaneschi \& Martins, 2002; Ribeiro, Omena \& Muricpy, 2003), siendo uno de los organismos más tolerantes a las variaciones de salinidad; en este sentido, se caracteriza por ser una especie básica dentro de las comunidades incrustantes encontradas en las raíces de mangle rojo, esto explicaría su presencia y abundancia. Los mayores valores 
de diversidad de organismos en las raíces de mangle se registraron en la zona norte, que aunque mostró la menor riqueza (33 especies) obtuvo una mayor equitabilidad, lo antes señalado puede estar relacionado con una mayor complejidad estructural o heterogeneidad de las raíces de mangle en la zona por su mayor desarrollo, ofreciendo más variedad de microhábitas potencialmente disponibles para los organismos en dicha zona.

En algunos estudios es difícil comparar zonas cuyas áreas de muestreos son diferentes, como es el caso de las raíces de mangle. Para solventar este problema Sanders (1968) propuso el método de Rarefacción para estimar el número de especies que existen en una muestra de $\mathrm{N}$ cantidad de organismos. El índice de rarefación de Sanders mensual, estimado para 1000 individuos, varió entre 13-27 especies, los valores más altos correspondieron a los meses de enero, julio y marzo; y los valores más bajos a los meses de septiembre y noviembre, coincidiendo, tanto los valores altos como los más bajos, con los resultados obtenidos con la diversidad de Shannon-Wiener. Con respecto a la dominancia, los bivalvos fueron más dominantes que los gasterópodos, lo cual podría explicarse porque los bivalvos son sésiles, sedentarios y filtradores mientras que los gasterópodos son móviles y más abundantes en zonas de Thalassia y corales (Prieto et al. 2003).

La estructura de la comunidad de bivalvos y gasteropodos de las raíces de mangle rojo por lo general es muy variable, tal y como se muestra en este estudio, no obstante, esta depende de muchos factores físicos, químicos, biológicos, fenología del manglar, presencia de ecosistemas vecinos (praderas de fanerógamas y arrecifes coralinos), exposición al oleaje, intensidad y dirección de las corrientes, luz incidente en las raíces (enterrada o sumergida), oferta larval y acción de los depredadores. En general, el ecosistema del mangle rojo $R$. mangle en isla Larga, presenta un número de bivalvos y gasterópodos superior al de otros ambientes de manglar ubicados en nuestras costas y el Caribe, esto puede deberse a las condiciones geográficas de isla Larga (ubicación) y las condiciones (temperatura, salinidad, oxígeno y nutrientes) presentes dentro de la bahía de Mochima, las cuales son favorables para el desarrollo y establecimiento de dichos organismos.

\section{AGRADECIMIENTOS}

Los autores desean expresar su agradecimiento al Instituto Nacional de Parques (INPARQUES), por el apoyo logístico y los permisos otorgados para realizar los muestreos en el Parque Nacional Mochima. A Deudedith Hernández y a María Elena Amaro, por la valiosa colaboración prestada en este estudio.

\section{RESUMEN}

Las raíces del mangle rojo forman un complejo ecosistema donde se reproducen, refugian y establecen de manera permanente una gran diversidad de organismos. En este estudio, se evaluó la diversidad de bivalvos y gasterópodos que habitan las raíces del mangle rojo Rhizophora mangle, en isla Larga, Bahía de Mochima, estado Sucre Venezuela. Se realizaron recolectas bimensuales desde enero 2007 hasta mayo 2008, en cuatro zonas de estudio denominadas: zona sur, norte, este y oeste. En cada zona se muestrearon cinco raíces de mangle, de donde se obtuvieron los especímenes. Se analizaron un total 180 raíces y se contabilizaron 35 especies de bivalvos y 25 de gasterópodos. Los bivalvos más abundantes fueron: Isognomon alatus, Isognomon bicolor, Ostrea equestris, Crassostrea rhizophorae y Brachidontes exustus, mientras que los gasterópodos más frecuentes: Littorina angulifera, Cymatium pileare y Diodora cayenensis. Los meses donde se observaron las mayores abundancias de especies y número de individuos fueron enero y julio 2007 y marzo 2008. El ecosistema de manglar en isla Larga, presentó un número de individuos y especies superior al reportado en otros trabajos realizados en Venezuela y otras regiones del Caribe.

Palabras clave: Parque Nacional Mochima, raíces de mangle, moluscos, Venezuela.

\section{REFERENCIAS}

Abbott, R. (1968). A guide to identification seashells of North America. Nueva York: Golden Press.

Abbott, R. (1974). American Seashells (2nd ed.). Nueva York: Van Nostrand Reinhold Co. 
Abbott, R., \& Morris, P. (1995). A field guide to shells Atlantic and gulf coasts and the West Indies. Peterson Field Guides. Boston, Massachusetts: Houghton Mifflin.

Amaro, M., \& Liñero-Arana, I. (2002). Demospongiae (Porifera) de isla Larga, bahía de Mochima, Venezuela. Boletín Instituto Oceanográfico de Venezuela, Universidad de Oriente, 41(1-2), 45-53.

Barreto, M. (2008). Diagnostics about the state of mangroves in Venezuela: Case studies from the National Park Morrocoy and Wildlife Refuge Cuare. In. Lieth, H.; García-Sucre, M., \& Herzog, B. (Eds.), Mangroves and Halophytes Restoration and Utilisation Series: Tasks for Vegetation Science, (Vol. 43, pp. 162). Springer Science.

Barrios, J., Márquez, M., \& Jiménez, M. (2003). Macroalgas asociadas a Rhizophora mangle L. en el golfo de Santa Fe, Estado Sucre, Venezuela. Boletín Instituto Oceanográfico de Venezuela, Universidad de Oriente, 42, 37-45.

Bendschneider, K., \& Robinson, R. (1952). A new spectrophotometric method for the determination of nitrite in sea water. Jornal Marine Research, 11, 87-97.

Cedeño, J., Jiménez, M., Pereda, L., \& Allen, T. (2010). Abundancia y riqueza de moluscos y crustáceos asociados a las raíces sumergidas del mangle rojo (Rhizophora mangle) en la laguna Bocaripo, Sucre, Venezuela. Revista Biologia Tropical, 58(3), 213-226.

Coelho, P., Tenório, D., Ramos-Porto, M., \& Mello, R. (2004). A fauna bêntica do estado de Pernambuco. In Eskinazi-Leça, E., Neumann-Leitão, S., \& Costa, M.F. (Eds.), Oceanografia: um cenário tropical (pp. 477-528). Bagaço, Recife.

Conde, J., \& Alarcón, C. (1993). Mangroves of Venezuela. Lacerda, L. D. (Ed.), Conservation and sustainable utilization of mangrove forests in the Latin and Africa Regions volume 2 of Part I. Latin America. Mangrove Ecosystems Technical Reports Series (pp. 211-243). Okinawa, Japan.

Conde, J., \& Suárez, C. (2003). Ecosistemas marino-costeros. In Aguilera, M., Azocar, A. \& González-Jiménez, E. (Eds.), Biodiversidad en Venezuela (pp. 863-883). Caracas, Venezuela: Fundación Polar.

Del Mónaco, C., Jiménez, E., Narciso, S., Alfonso, F., \& Bustillos, F. (2010). Caracterización de los bosques de manglar y las praderas de Thalassia testudinum de la isla la Tortuga y cayos adyacentes, Venezuela. Boletín Centro Investigaciones Biológica, Universidad del Zulia, 44(3), 297-316.

Díaz, J., \& Puyana, M. (1994). Moluscos Marinos del Caribe Colombiano: un Catálogo Ilustrado. ColcienciasFundación Natura Colombia-Invemar, Bogotá

Domaneschi, O., \& Martins, C. (2002). Isognomom bicolor (CB. Adams) (Bivalvia Isognomidea): primero registro para o Brasil descrição de espécie e considerações sobre a ocorrência e distribuição de Isognomom na costa brasileira. Revista Brasileira de Zoología, 19(2), 611-627.

FAO. (2007). The world's mangroves 1980-2005. FAO Forestry Paper No. 153. Roma, Lazio, Italia.

Guerra-Castro, E., Cruz-Motta, J., \& Conde, J. (2011). Cuantificación de la diversidad de especies incrustantes asociadas a las raíces de Rhizophora mangle $\mathrm{L}$. en el Parque Nacional laguna de la Restinga. Interciencia, 36 (12), 923-930.

Herrera, L., \& Febres, G. (1975). Proceso de sugerencia y renovación en la Fosa de Cariaco. Mar Caribe. Boletín Instituto Oceanográfico de Venezuela, Universidad de Oriente, 14 (1), 31-44.

Jiménez, M., \& Liñero-Arana, I. (2002). Moluscos en praderas de Thalassia testudinum en isla larga, bahía de Mochima, edo. Sucre, Venezuela. Boletín Instituto Oceanográfico de Venezuela, Universidad de Oriente, 41 (1-2), 55-65.

Kathiresan, K., \& Binghan, B. (2001). Biology of mangroves and mangrove ecosystems. Advances in Marine Biology, 40, 81-251.

Koroleff, F. (1969). Determination of ammonia. In Grasshof, K., Ehrhardt, M., \& Kremling, K. (Eds.), Methods of Seawater Analysis (pp. 419). Weinheim, Alemania: Verlag Chemie.

Krebs, C. (1985). The experimental analysis of distribution and abundance (3er ed.). Nueva York: Harper and Row.

Krebs, C. (1989). Ecological methodology. Nueva York: Harper Collins Publishers.

Lacerda, L., Conde, J., Kjerfve, B., Álvarez-León, R., Alarcón, C., \& Polanía, J. (2001). American mangroves. Lacerda, L.D. (Ed.). In Mangrove Ecosystem: Function and Management (pp. 1-62). Berlín, Alemania: Springer.

Lee, S. (2008). Mangrove macrobenthos: Assemblages, services and linkges. Journal Sea Research 59, 16-29.

Lodeiros, C., Marín, B., \& Prieto, A. (1999). Catálogo de moluscos marinos de las costas nororientales de Venezuela: Clase Bivalvia. Cumaná, Sucre, Venezuela: Edición APUDONS.

López, B., Barreto, M., \& Conde, J. (2011). Cuantificación de la diversidad de especies incrustantes asociadas a las raíces de Rhizophora mangle L. en el Parque Nacional laguna de la Restinga. Interciencia, 36 (12), 923-930.

Marcano, B. (1996). Fijación y crecimiento de Botryllus planus (Van Name, 19-2) ascidia colonial, en raices sumergidas de Rhizophora mangle (L.) en la ensenada de Turpialito, Estado Sucre, Venezuela. (Trabajo de grado). Universidad de Oriente, Cumaná, Venezuela.

Margalef, R. (1995). Ecología. Barcelona, España: Ediciones Omega, S.A.

Márquez, B., \& Jiménez, M. (2002). Moluscos asociados a las raíces sumergidas del mangle rojo Rhizophora 
mangle, en el Golfo de Santa Fe, Estado de Sucre, Venezuela. Revista Biología Tropical, 50(3-4), 1101-1112.

Márquez-Rojas, B., Blanco-Rabla, J., Jiménez, M., \& Allen, T. (2006). Crustáceos asociados a las raíces del mangle rojo Rhizophora mangle (L.) en el golfo de Santa Fe, Estado Sucre, Venezuela. Ciencia, 14, 12-27.

Medina, E., \& Barboza, F. (2003). Manglares del sistema del lago de Maracaibo: Caracterización fisiográfica y ecológica. Ecotrópicos, 16(2), 75-82.

Montes, A. (1992). Estudio ecológico de las comunidades de ascidias incrustadas en raices de Rhizophora mangle en dos localidades del Estado Sucre, Venezuela. (Trabajo de Ascenso). Universidad de Oriente, Cumaná, Venezuela.

Morao, A. (1983). Diversidad y fauna de moluscos y crustáceos asociados a las raíces sumergidas del mangle rojo, Rhizophora mangle en la Laguna de la Restinga. (Tesis de pregrado). Universidad de Oriente, Isla de Margarita, Venezuela.

Moreno-Ríos, C. (2007). Moluscos y crustáceos asociados a las raices sumergidas del mangle rojo, Rhizophora mangle (L.1753) y su relación frente a la calidad del agua en dos aéreas de la Bahía de Cispata "Cienagas del Ostional y Navio y Ciénaga de la Soledad" (Córdoba), Caribe Colombiano. (Trabajo de grado). Universidad de Bogotá Jorge Tadeo Lozano, Bogotá.

Murillo, P. (2004). Composición taxonómica y proceso sucesional de la fauna asociada a las raices sumergidas de Rhizophora mangle (Mangle rojo) de san Andrés isla. (Trabajo de pregrado en Biología Marina). Universidad Nacional de Colombia, Colombia.

Murphy, J., \& Riley, J. (1962). A modified single solution method for the determination of phosphate in natural waters. Analytica Chimica Acta, 27, 31-36.

Okuda, T. (1975). Características hidroquímicas del golfo de Santa Fe y aéreas adyacentes. Boletín Instituto Oceanográfico de Venezuela, Universidad de Oriente, 14(2), 251-268.

Ordosgoitti, R. (1985). Estudio ecológico de la epifauna en raices sumergidas del mangle Rhizophora mangle en la bahía de Mochima (Tesis de pregrado en Biología Marina). Universidad de Oriente, Cumana, Venezuela.

Pico, E., Hernández-Rivas, O., \& Holguín-Quiñones, O. (2008). The zonation and density of the macrimolluks living in the mangrove swamps of the sand barrier of el mogote, la Paz, Baja California Sur. In 42 th Annual Meeting of the Western Society of Malacologists-California State University, Fullerton (CSUF). Libro de resumen California-U.S.A.

Pomareda, E., \& Zanella, I. (2006). Diversidad de moluscos asociados a manglares en isla San Lucas. Revista Ambiente, 30, 11-13.

Portocarrero, M. (2004). Estudio de la comunidad de moluscos epibiontes sobre raices sumergidas del mangle rojo (Rhizophora mangle) en Isla Barú. Caribe colombiano. (Trabajo de pregrado). Universidad Nacional de Colombia, Colombia.

Prieto, A., Sant, S., Méndez, E., \& Lodeiros, C. (2003). Diversidad y abundancia de moluscos en las praderas de Thalassia testudinum de la Bahía de Mochima, Parque Nacional Mochima, Venezuela. Revista Biología Tropical, 51(2), 413-426

Quintero, A., Bonilla, J., Serrano, L., Amaro, M., Rodríguez, B., Terejova, G., \& Figueroa, Y. (2004). Características ambientales de la bahía de Mochima y adyacencias de la cuenca de Cariaco, Boletín Instituto Oceanográfico de Venezuela, Universidad de Oriente Venezuela, 43(1-2), 49-64.

Ribeiro, S., Omena, E., \& Muricpy, G. (2003). Macrofauna associated to Mycle microsigmatasa (Porifera, Demospongiae) in Rio de Janiero State, SE Brazil. Estuarine, Coastal and Shelf Science, 57(5-6), 951-959.

Sánchez-Arias, L., Paolini, J., \& Rodríguez, J. (2010). Dinámica de las propiedades del suelo en bosques de Rhizophora mangle L. (Rhizophoraceae) en Isla de Margarita, Venezuela. Revista Biología Tropical, 58 (2), 547-564.

Sanders, H. (1968). Marine benthic diversity: A comparative study. American Naturalist, 102(925), 243-282.

Steel, R., \& Torrie, J. (1960). Bioestadística: principios y procedimientos. (2nd ed.). México: Prentice-Hall-Hispanoamérica.

Strickland, J., \& Parsons, H. (1972). A Practical Handbook of Seawater Analysis. (2nd ed.). Bulletin of the Fisheries Research Board of Canada, 167, 310.

Treguer, P., \& Le Corre, P. (1975). Manual d'analyses des sels nutritifs dans léau de mer (2nd ed.). Utilization de I' Auto-Analyzer II Technicon. Brent, Francia: LOC-UBO.

Valle, A. (2007). Estructura del bosque de manglar y algunos aspectos sobre la fauna asociada a las raíces de Rhizophora mangle (L., 1773) en la ciénaga de Cholón, Isla Barú, municipio de Cartagena, Caribe colombiano. In Informe del Estado de Los Recursos Marinos y Costeros Año 2006 (pp. 237-240). Series de Poblaciones Especies.

Villafranca, S. (2007). Malacofauna en raices sumergidas del mangle Rhizophora mangle en la laguna hipersalina de La Restinga, isla de Margarita, estado Nueva Esparta, Venezuela. (Trabajo de Ascenso). Universidad de Oriente, Cumaná, Venezuela.

Warmke, M., \& Abbott, R. (1975). Caribbean seashells. A guide to the marine molluscs of Puerto Rico and other West Indian Islands, Bermuda and the Lower Florida Keys. Nueva Jersey. 
